\title{
Effector Recognition and Activation of the Arabidopsis thaliana NLR Innate Immune Receptors
}

\author{
A.D. Steinbrenner, S. Goritschnig, K.V. Krasileva, K.J. Schreiber, \\ AND B.J. STASKAWICZ \\ Department of Plant and Microbial Biology, University of California, Berkeley 94703 \\ Correspondence: stask@berkeley.edu
}

\begin{abstract}
Plant immunity to coevolved pathogens relies on the perception of pathogenic effectors by nucleotide-binding domain and leucine-rich repeat-containing (NLR) proteins - sophisticated intracellular receptors that have both perception and signaling roles in activating defenses. Given the conserved domain architecture of NLRs, a structural biology perspective is particularly relevant to understanding mechanisms of their activation. Here, we summarize our recent findings on the Arabidopsis resistance protein RPP1, a member of the TIR-NBS-LRR family of plant NLRs that specifically recognizes the cognate effector protein ATR1. To study the basis of RPP1 activation, we have taken advantage of a series of RPP1 and ATR1 alleles that differentially condition resistance. In planta association between the LRR domain of RPP1 and ATR1 only occurs in a resistance-activating combination of alleles, suggesting that a direct interaction between RPP1 and ATR1 protein leads to activation of the NLR (Krasileva et al. 2010). Given critical amino acid residues' locations on the solved ATR1 crystal structure, and variable amino acid residues on the predicted LRR structure of RPP1, we hypothesize that specific "hotspots" of the horseshoe-like LRR fold mediate binding to the ATR1 ligand and that polymorphisms mapping to these surfaces condition differences in allelic recognition specificity. We present docking models of a possible co-complex between RPP1 and ATR1, and we propose that ATR1 binding relieves autoinhibition of RPP1 resistance signaling. This is consistent with current models of activation for both TIR- and CC-type plant NLRs, where conformational changes could lead to NLR oligomerization, nucleotide binding, translocation, and other critical downstream events in triggering immunity.
\end{abstract}

Plants possess a cell-autonomous multilayered immune system that allows them to successfully defend against most attacking microbial pathogens (Dodds and Rathjen 2010). The primary immune response involves the extracellular recognition of conserved pathogen-associated microbial patterns (PAMPs) by pattern recognition receptors (PRRs) (Boller and Felix 2009). Adapted pathogens have evolved to deploy arsenals of effector proteins to the plant to subvert this basal immunity and thus enhance pathogen virulence (Cunnac et al. 2011). Plants in turn evolved mechanisms to recognize the presence of effectors and to mount an effective resistance response (Chisholm et al. 2006; Jones and Dangl 2006). The interface of this continuous evolutionary battle is the interaction of innate immune receptors with their cognate, recognized effector proteins, forming the basis of highly specific, gene-for-gene recognition among various plant and pathogen genotypes.

Recognition of pathogen signatures involves receptor proteins that typically contain a series of leucine-rich repeats (LRRs). Extracellular LRRs form the recognition domains of receptor-like kinases such as the PRRs flagellin sensing 2 (FLS2), ef-Tu receptor (EFR), or Xa21, which recognize the bacterial PAMPs flagellin, elongation factor Tu, or sulfated Ax21, respectively (Chinchilla et al. 2006; Zipfel et al. 2006; Lee et al. 2009). Intracellular effector recognition is accomplished by a family of cytosolic nucleotide-binding site (NBS)-LRR proteins (NLRs) that share structural and functional homology with nucleotide-binding oligomerization domain (NOD)like receptors in mammalian innate immunity (Inohara et al. 2005). The carboxy-terminal LRR domain is involved in protein-protein interaction and typically confers effector recognition specificity (Farnham and Baulcombe 2006; Sela et al. 2012). NLR activation after effector recognition requires nucleotide exchange at the NBS domain (Lukasik and Takken 2009). Amino-terminal domains are predicted to be involved in downstream signaling after NLR activation, but precise mechanisms have yet to be elucidated. Because effector recognition by plant NLRs typically triggers a rapid cell death of the infected and surrounding cells, their activation must be tightly regulated, initiating signaling quickly and specifically only in response to signatures of attack by coevolved pathogens. It is thus likely that the multiple domains of NLRs and their intramolecular interactions reflect these needs. Current models postulate negative regulation of amino-terminal signaling and NBS-domainmediated nucleotide hydrolysis via intramolecular binding to the LRR domain (Takken and Goverse 2012). This would allow for a steady-state expression level of a negatively regulated receptor protein able to be quickly activated after effector recognition. Understanding the exact molecular mechanisms of this critical event - the switch from an "off" to "on" state - is an important area of research in plant innate immunity.

The modes of effector recognition of plant NLR immune receptors can be as diverse as their targets. So far, 
three main models of effector recognition have emerged. The guard hypothesis describes host protein targets of pathogen effectors that are monitored by NLRs for pathogen-induced modifications, such as phosphorylation or proteolysis. The best-studied example for this model is the Arabidopsis protein RIN4, which is targeted by a number of bacterial effectors including AvrRpt2 and AvrRpm1 and is monitored by two NLRs in Arabidopsis: RPM1 and RPS2 (Mackey et al. 2002; Day et al. 2005; Kim et al. 2005). This indirect recognition model allows for the maintenance of highly effective NLRs, because they detect a fitness-enhancing virulence activity of pathogen effectors that is favorably maintained in the pathogen population. Some effector targets are proposed to act as "decoys" rather than actual virulence targets, mimicking functional proteins. An example for this interaction is provided by tomato Pto, which mimics the kinase domains of PRRs, the actual virulence targets of AvrPto and AvrPtoB (Oh and Martin 2011). When the effector phosphorylates its target proteins including Pto, the associated NBS-LRR Prf becomes activated and triggers resistance. Finally, a number of NLRs have been shown to interact with and directly recognize their cognate effector, consistent with a ligand-receptor model of activation. Examples for this interaction include the fungal effectors AvrPita, AvrL567, and AvrM, their corresponding NLRs Pita, L, and M, as well as the oomycete effector ATR1, which is recognized by the Arabidopsis NLR RPP1 (Jia et al. 2000; Dodds et al. 2006; Catanzariti et al. 2009; Krasileva et al. 2010). In an evolutionary context, this "arms race" interaction often results in highly polymorphic loci for both the pathogen effector and host NLR receptor.

In this report, we summarize and review our findings about the molecular interaction between the RPP1 resistance protein from Arabidopsis thaliana and its cognate effector ATR1 from Hyaloperonospora arabidopsidis $(\mathrm{Hpa})$, including mounting evidence for direct interaction between the NLR and effector. We have used surrogate expression systems, structural analysis, computer modeling, and mutational analyses to study the interaction between RPP1 and ATR1. We present a model of RPP1 activation by ATR1 and discuss how our findings will inform future studies regarding NLR activation and function.

\section{SURROGATE SYSTEMS TO STUDY BIOTROPHIC INTERACTIONS}

The Arabidopsis / Hpa pathosystem has emerged as an excellent model to study plant disease resistance, despite the limitations imposed by the biotrophic lifestyle of this oomycete pathogen. Several NLR genes conferring Hpa resistance have been identified, and the recent sequencing of the genome has enabled computational predictions of putative effectors (Cabral et al. 2011; Fabro et al. 2011; Goritschnig et al. 2012). In contrast to the related hemibiotrophic oomycete Phytophthora infestans, the effector repertoire of Hpa is greatly reduced and shows signs of adaptation to its biotrophic lifestyle (Baxter et al. 2010). To dissect the contributions of individual Hpa effectors and more precisely study their cognate NLRs, several surrogate expression systems have been developed, including particle bombardment, Agrobacterium-mediated transient expression, and delivery via viral vectors or the type-three-secretion-system (TTSS) of plant-pathogenic Pseudomonas species (Sohn et al. 2007; Rentel et al. 2008; Kale and Tyler 2011). Interpretation of virulence contributions of individual effectors in these assays is not straightforward. Transgenic Arabidopsis plants stably overexpressing several Hpa effectors were shown to exhibit increased susceptibility to Hpa infection (Fabro et al. 2011). However, in planta overexpression of individual effectors may not lead to biologically relevant concentrations and will mask potential pathogen-mediated posttranslational modifications. Bacterial delivery of the Hpa effectors ATR1 and ATR13 was reported to increase bacterial virulence by Sohn et al. (2007), but we were unable to reproduce the result (Krasileva et al. 2011; Leonelli et al. 2011). It is conceivable that effectors are acting in a concerted fashion to exert virulence function or may only have virulence roles in a natural Hpa infection-for example, in initiating and maintaining a haustorial interface with the plant cell. In contrast with analysis of effector virulence functions, surrogate assays have proven to be useful for investigating effector recognition events by plant NLRs. Screening effector libraries from $P$. infestans and Hpa against different accessions of their host plants has led to the identification of novel recognition specificities (Vleeshouwers et al. 2008; Goritschnig et al. 2012). Transient expression assays in tobacco have been instrumental in dissecting effector recognition events, because successful recognition is easily visualized as macroscopic cell death (the hypersensitive response, or HR) when a recognizing NLR protein is coexpressed with its cognate effector.

\section{ALLELIC SERIES OF ATR1 AND RPP1}

The foundations for studying the specific ATR1 and RPP1 interaction were laid in the 1990s after cloning of the first RPP (recognition of Peronospora parasitica, now known as $\mathrm{Hpa}$ ) resistance genes conferring Hpa resistance (Holub and Beynon 1997; Parker et al. 1997). RPP1-WsB was isolated from the Wassilewskija (Ws) ecotype in a locus containing three paralogs using positional cloning (Botella et al. 1998). Later efforts isolated a functional RPP1 gene in the RPPI locus of the Niederzenz (Nd) accession, which contains nine paralogs (Gordon 2002). The locus appears to have multiple recognition capacities, because RPP1 paralogs A and C in Ws also confer resistance to certain Hpa strains through recognition of as yet unidentified effectors (Botella et al. 1998). RPP1-WsB and RPP1-NdA belong to the Toll/ interleukin receptor/R gene (TIR) class of NBS-LRR proteins, containing an amino-terminal TIR domain that have homology with signaling domains in animal Tolllike receptors. The corresponding Hpa effector ATRl 
(A. thaliana recognized 1) was identified in genetic crosses of virulent and avirulent Hpa strains (Gunn et al. 2002). ATR1 contains an amino-terminal eukaryotic secretion sequence followed by the host-translocation motif Arg$\mathrm{X}$-Leu-Arg and an effector domain. The effector domain of ATR1 is highly polymorphic in different $H p a$ strains, and a series of ATR1 alleles was used to demonstrate a spectrum of recognition capacity, as determined by bombardment assays of individual effectors in resistant and susceptible Arabidopsis (Rehmany et al. 2005). The allelic variation present in both RPP1 and ATR1 provides an excellent tool set for studying effector recognition events. In addition to available RPP1 alleles, we recently found four additional Arabidopsis accessions (Pu2-23, Est-1, Ws-2, Zdr-1) capable of recognizing different alleles of ATR1 (Krasileva et al. 2011). Initial complementation tests indicate that the responsible loci are allelic with RPP1 from Ws-0 and Nd-1, potentially expanding the allelic series of RPP1 (S Goritschnig, unpubl.).

Using effector domain constructs of ATR1 (excluding the eukaryotic secretion sequence and host translocation motif) expressed by Agrobacterium, the allelic spectrum of ATR1 recognition by RPP1-NdA and RPP1-WsB was recapitulated and extended in transient tobacco assays (Fig. 1A) (see also Fig. 1A of Krasileva et al. 2010). Of all ATR1 alleles tested, RPP1-NdA recognizes only ATR1-Emoy2, whereas RPP1-WsB has a greater recognition competence and triggers a defense response to ATR1-Emoy2, ATR1-Emco5, and ATR1-Maks9. Neither RPP1 allele is able to recognize ATR1-Cala2, providing a spectrum of differential recognition specificities between the two RPP1 alleles.

\section{RECOGNITION CORRELATES WITH INTERACTION BETWEEN THE NLR AND EFFECTOR}

Using this series of differentially recognizing effector and NLR alleles, subsequent work in our laboratory has helped to elucidate the molecular mechanism of RPP1 activation. The degree of polymorphism among both the RPP1 and ATR1 alleles, as well as the presence of allelespecific recognition, suggested a potential direct interaction between the two proteins. Using epitope-tagged ATR1 and RPP1 in coimmunoprecipitation experiments, the NLR and effector were shown to associate in planta. The ability of ATR1 to associate with RPP1 in transient expression assays correlated with recognition activity across several ATR1 alleles (Krasileva et al. 2010). Recognition-specific association is consistent with a ligand-receptor model of plant NLR activation and suggests direct interaction of the two proteins. Using RPP1 deletion constructs in coexpression assays determined that only the LRR domain associated with ATR1, consistent with the effector binding to this domain. In addition, mutations in the RPP1 TIR domain or NBS P-loop affecting downstream signaling did not abolish interaction with the recognized effector, indicating that effector binding happens before NLR protein activation (Krasileva et al. 2010).

LRR association with ATR1 is compatible with theoretical and experimental studies of the domain's role in the function of NLR immune receptors. Polymorphism among NLR alleles is often highest in LRRs, and thus, it has long been postulated that variation in effector recognition should map to this domain. This has been validated
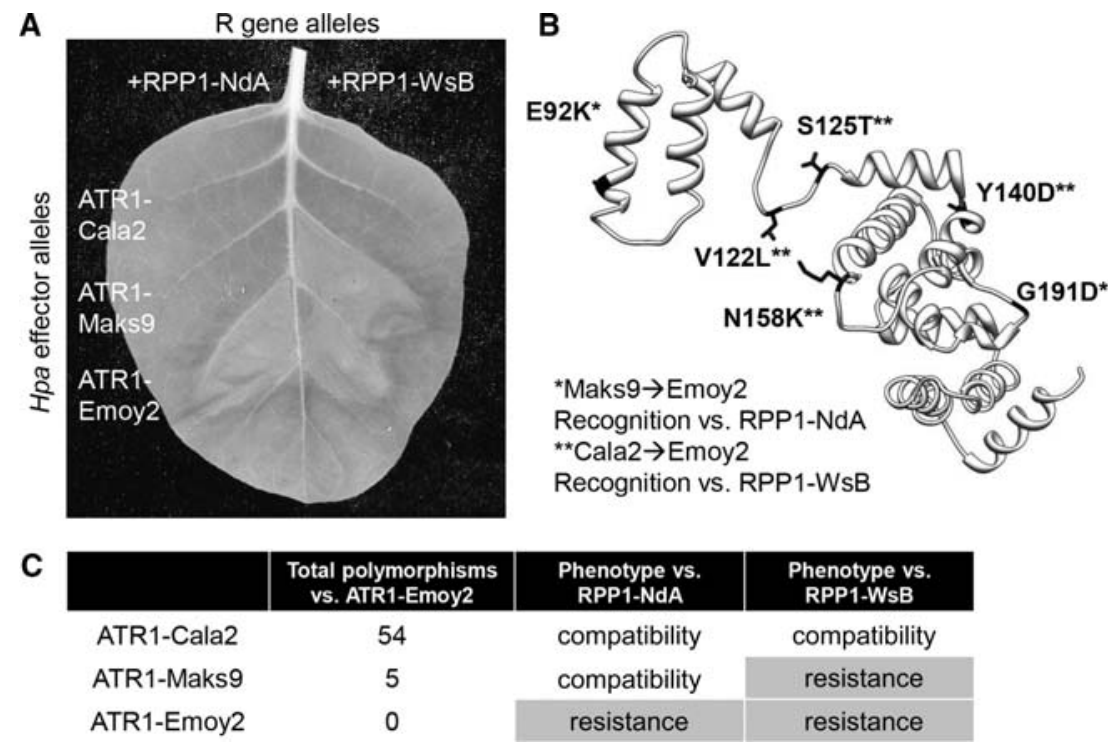

Figure 1. Recognition spectra of functional RPP1 alleles from two accessions against ATR1 alleles from three $H p a$ strains. $(A)$ Surrogate assay for resistance/compatibility by screening hypersensitive response (HR) phenotypes of allelic combinations. Nicotiana tabacum plants were inoculated with Agrobacterium strains carrying plasmids to express either RPP1 or ATR1. (B) Solved crystal structure of ATR1-Emoy2 with critical residues highlighted. Two substitutions (single asterisk) were sufficient to impart recognition to ATR1-Maks9 by RPP1-NdA, whereas four different substitutions (two asterisks) imparted recognition to ATR1-Cala2 by RPP1-WsB. (C) Summary of resistance spectra and amino acid sequence comparison of three ATR1 alleles. 
with experimental data for NLRs from multiple plant species including flax, rice, and tomato (Ellis et al. 1999; Jia et al. 2000; Van der Hoorn et al. 2001).

\section{STRUCTURAL INSIGHT INTO MOLECULAR RECOGNITION OF ATR1 BY RPP1}

Despite the correlation of ATR1-RPP1 complex formation with RPP1 activation as well as the implication of the RPP1 LRR domain in recognition, the exact structural basis of RPP1 activation remains unclear. By analyzing allelic variation in the context of structural data and models, it should be possible to predict the interacting surfaces on both RPP1 and ATR1 and model the potential for NLR activation by conformational changes among domains.

The first experiments toward this end took advantage of polymorphisms among ATR1 alleles. ATR1-Emoy2 and ATR1-Maks9 differ by only five amino acid polymorphisms yet are differentially recognized by RPP1-NdA. Individually substituting these Emoy2 amino acids in a Maks9 background revealed that introducing Lys92 or Gly191 fully reconstitutes the capacity to trigger the HR in tobacco (Krasileva et al. 2010). A similar approach was taken using a second unrecognized allele, ATR1-Cala2. Despite a much lower $82 \%$ amino acid similarity between ATR1-Cala2 and the recognized allele ATR1-Emoy2, potentially important polymorphisms were identified by narrowing down candidate residues to those amino acids conserved in all ATR1 alleles recognized by RPP1-WsB. Of these 14 Cala $2 \rightarrow$ Emoy 2 amino acid substitutions, four were sufficient to impart weak recognition capability by RPP1-WsB in an ATR1-Cala background (Chou et al. 2011). The effects of these mutations were quantitative and additive, because substituting all four residues recapitulated a near wild-type-like HR. Interestingly, these substitutions only affected recognition by RPP1-WsB, and mutants remained unrecognized by RPP1-NdA. These critical residues are highlighted on the recently solved ATR1 crystal structure in Figure 1B. Notably, all but one of these amino acid residues lie on the surface of the ATR 1 protein, consistent with their importance in proteinprotein interaction with the RPP1 LRR.

Several conclusions can be drawn from these findings. First, knowledge regarding natural variation in effectors can be used to quickly gain insight into the basis of recognition, because a spectrum of alleles and recognition phenotypes allows for homology-based assessment of the relative importance of amino acid polymorphisms. Second, two functional RPP1 alleles, RPP1-NdA and RPP1WsB, can recognize distinct surfaces of ATR1, because the gain-of-recognition mutations in ATR1 map to different protein surfaces on the ATR1 crystal structure. Third, NLR immune receptor activation may be quantitative and additive, as demonstrated by the weak gain-of-recognition mutants of ATR1-Cala2 and increasing activation of recognition when combined.

We are performing further work on the molecular basis of ATR1 activation of resistance. Our preliminary data indicate that novel gain-of-recognition mutants of ATR1-Cala2 are recognized only by RPP1-NdA, and not RPP1-WsB, as opposed to those described above (A Steinbrenner, unpubl.). Thus, different RPP1 alleles can have distinct, nonoverlapping recognition capabilities (i.e., RPP1-WsB is not simply a more sensitive receptor than RPP1-NdA). Assuming that HR activation correlates with coimmunoprecipitation of RPP1 for these mutant alleles, this is further evidence that RPP1 acts as a sophisticated and highly specific intracellular immune receptor.

\section{STRUCTURAL MODELING OF LRR INTERACTION SURFACES}

The genetic and biochemical data described above are consistent with a direct effector-R protein interaction, with ATR1 potentially binding the surface of the RPP1 LRR. More general structural findings regarding LRR domains as ligand-interacting modules (Bella et al. 2008) may thus provide insight into the basis of ATR1 recognition. Solved crystal structures of LRR-containing proteins all indicate a horseshoe-like structure, with each motif repeat composing one turn in a solenoid-like fold that exposes a large overall surface area (Kobe and Deisenhofer 1993; Di Matteo et al. 2003). Each repeat typically contains one $\beta$ strand in a continuous $\beta$ sheet, providing a platform for ligand binding. For example, the protein RNase inhibitor (RNI), consisting entirely of leucine-rich repeats, has the highest ligand-binding affinity of any known biological proteinprotein interaction in its interaction with RNase analogs (Dickson et al. 2005). We predict that the functionally important residues in RPP1 mediating ATR1 binding will map to the surface of a similar LRR fold and that allelic variation in this domain can be used to study the basis for differential ATR1 recognition. The domain is highly variable between RPP1-NdA and WsB (the two alleles share $84 \%$ amino acid similarity, including multiple indels), and many polymorphic residues map to the surface of the LRR when the domain's structure is modeled using the homology server Phyre (Fig. 2A).

Are these surface polymorphisms the determinants of ATR1 recognition? Such a finding would be consistent with several other studies of LRR interaction with ligands. In one recent example, Helft et al. (2011) developed the software package "Repeat Conservation Mapping" (RCM) to map highly variable patches on LRRs. Double alanine substitutions in the well-characterized LRR-containing PRRs EFR and FLS2 established a correlation between patches of amino acid conservation and the ability to respond to their respective ligands, elf18 and flg22. Thus, key surfaces for receptors binding to highly stable targets, such as elongation factor Tu and flagellin, can be predicted by amino acid conservation. Inversely, patches of variability in RPP1 may represent the basis for differential ligand binding (i.e., different alleles of ATR1).

On the basis of evidence from other LRR-containing proteins, we predict that recognition specificity will be determined by amino acids on the concave $\beta$ sheet of RPP1. Many of the polymorphisms between RPP1-NdA 


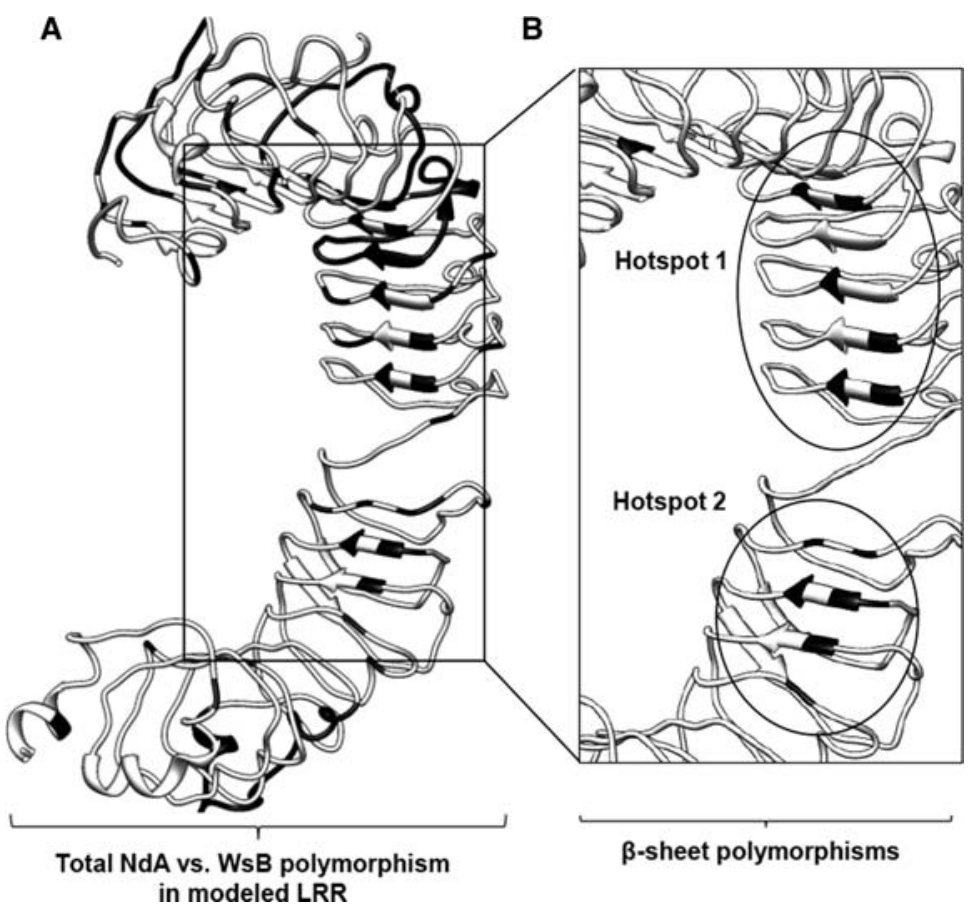

Figure 2. Polymorphisms in the modeled LRR of RPP1. (A) Predicted structure of the RPP1-WsB LRR domain. The primary amino acid sequence of RPP1-WsB downstream from the NB-ARC domain was submitted to the structural prediction server Phyre. The top confidence model was against the solved crystal structure of human TLR3. Polymorphisms and insertions in RPP1-WsB relative to RPP1-NdA are highlighted in black. ( $B$ ) Circled are hotspots of polymorphism that map to the semicontinuous $\beta$ sheet of the concave surface of the horseshoe-like LRR fold.

and RPP1-WsB map to this surface in the LRR structure (Fig. 2B). Interestingly, two "hotspots," with many polymorphisms occurring on adjacent $\beta$ strands, are apparent in different regions of the LRR. If patches of hypervariability provide a platform for differential binding to a ligand, we hypothesize that these hotspots could determine differential binding affinity to ATR1 alleles. Interestingly, these polymorphisms occur in a similar orientation (i.e., multiple amino acids on adjacent $\beta$ strands) to known binding determinants in the canonical LRR-ligand interaction RNI bound to RNase analogs (Johnson et al. 2007). Kinetic studies of RNI identified several hotspots of binding by mutating surface contacting amino acid residues observed in the co-crystal structure. All of these critical residues lie on the concave surface of RNI and occur across multiple, adjacent $\beta$ strands.

Current experiments in the laboratory are testing these hypotheses using mutagenesis and validation of biochemical and biological functionality of mutant NLRs. Regardless of the importance of these hotspots, our approach demonstrates the power of combining knowledge of both natural variation and protein structure to generate hypotheses regarding the basis of NLR specificity.

\section{PREDICTED COCOMPLEXES OF RPP1 AND ATR1}

An in silico analysis of the protein-protein interaction may also provide hypotheses regarding ATR1-RPP1 interaction. To investigate the potential for a ligand-receptor interaction of ATR1 and RPP1, we performed docking simulations of the ATR1 crystal structure with the RPP1 LRR homology model using the protein-protein complex prediction server ZDOCK (http://zdock.umassmed.edu; Chen et al. 2003). The server outputs up to 2000 predicted orientations of a potential complex given two PDB structures. We used the server to dock the ATR1-Emoy2 crystal structure against the modeled RPP1-WsB LRR. Whereas the orientation of ATR1 varied greatly in the top predicted complexes, the vast majority of surface contact points always lay on the concave surface of the putative RPP1 $\beta$ sheet, consistent with findings for other ligand-binding LRRs. A model of a representative complex is depicted in Figure 3A. Interestingly, many of the predicted complexes show hydrogen bonding between polymorphic residues of both proteins. For example, in the representative structure, polymorphic amino acid residues from "hotspot 2," depicted in red, are contacting a surface of ATR1 that contains a critical residue for RPP1NdA recognition (Gly191 in Fig. 3A). Close positioning of polymorphic residues in a putative protein-protein complex could reflect the history of molecular coevolution between the two proteins: both effector evasion of detection via mutation and redetection by mutated NLR alleles better able to interact with the effector ligand.

The ZDOCK server also allows for constraining the model based on genetic data by forcing specific residues to be in or out of contact. We constrained complexes to force contact between the LRR surface and one of two 

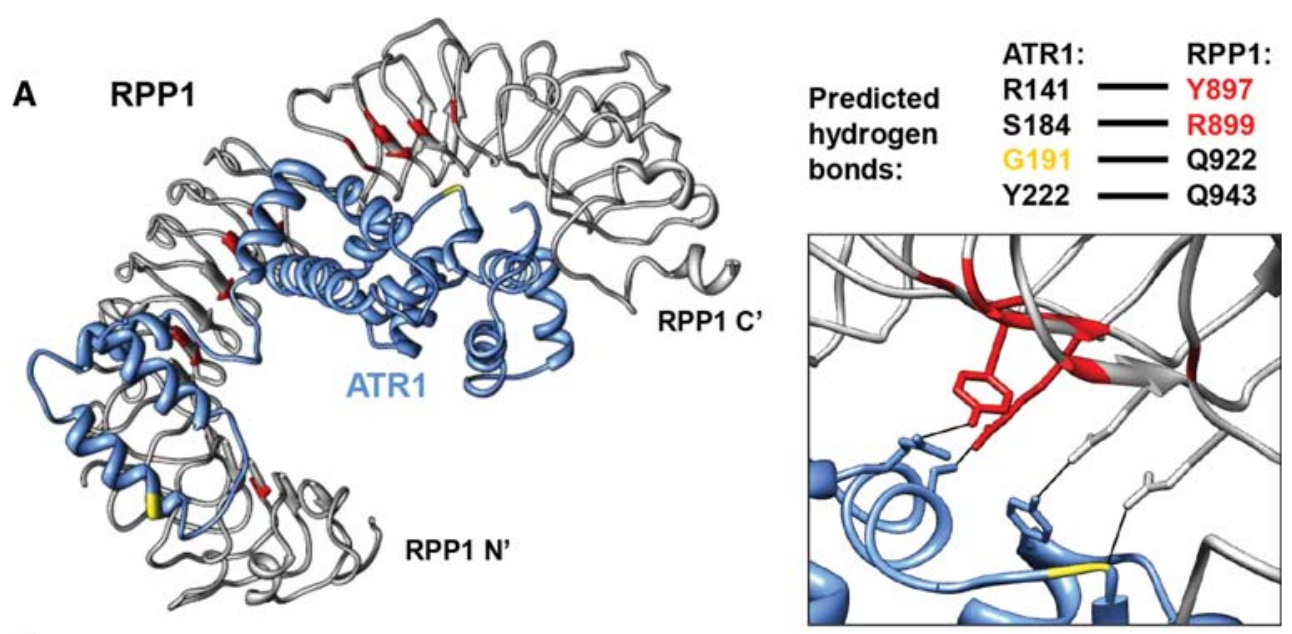

B
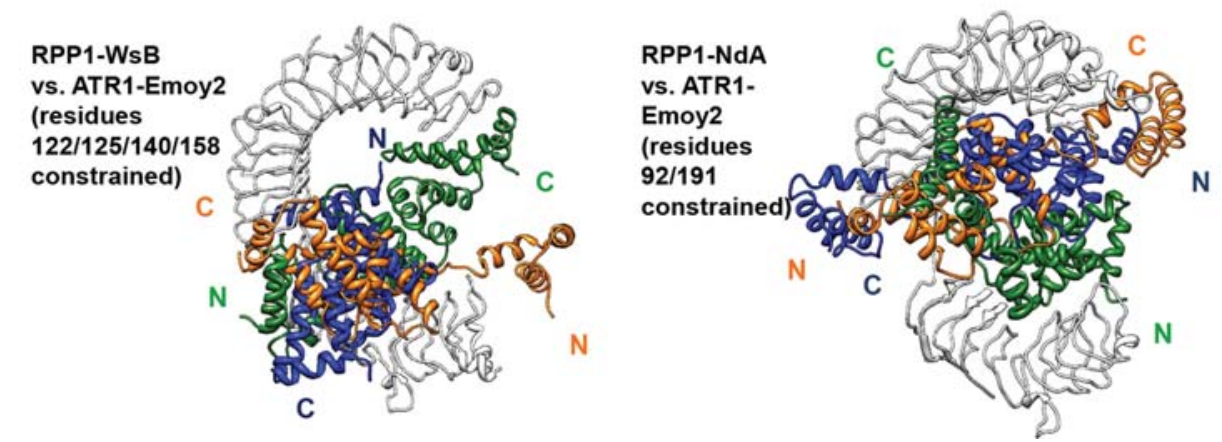

Figure 3. Docking models of RPP1 and ATR1. (A) A representative predicted complex showing the modeled RPP1-WsB LRR (white) and the ATR1-Emoy2 crystal structure (blue). (Red) $\beta$-sheet polymorphisms in RPP1, (yellow) ATR1 residues critical for RPP1 recognition. (Right) Several predicted hydrogen bonds among polymorphic residues. $(B)$ Constrained docking simulations excluding models that do not have genetically determined critical amino acids of ATR1 in contact with RPP1. The top three complexes are shown in different colors, with RPP1 in the same orientation. Indicated are amino- and carboxy-termini of each predicted ATR1 orientation.

sets of ATR amino acids: either Lys92/Gly191 or Leu122/Thr125/Asp140/Lys158 in ATR1, respectively. Because these sets of amino acids are sufficient to confer RPP1 recognition to unrecognized ATR1 alleles (ATR1Maks9 and ATR1-Cala2, respectively), we reasoned that they are most likely to be in contact in a recognizing interaction, given a ligand-receptor model of effector recognition. Contact filtering greatly narrowed potential complexes, from 2000 modeled complexes to just 14 that met the first set of amino acid contact criteria (using the RPP1-NdA-predicted LRR) and three that met the second criteria (using the RPP1-WsB-predicted LRR). Surprisingly, even these constrained models varied greatly in ATR1 orientation relative to the LRR (see top three models in Fig. 3B), indicating that many possible binding orientations are energetically favorable.

It is also notable that modeled LRR complexes with the predicted RPP1 TIR domain (modeled to the solved crystal structure of the flax NLR L6 [Bernoux et al. 2011]) also placed the TIR domain in surface contact with the concave $\beta$-sheet surface. This is consistent with intramolecular negative regulation of the TIR domain by the LRR and NLR activation via ligand replacement with a new, higher-affinity intermolecular interaction with ATR1. We emphasize that these predicted complexes are only models, but they are nonetheless helpful to illustrate potential mechanisms of RPP1 activation via ATR1 recognition. Ultimately, the variability in docking models suggests that it will be necessary to purify and crystallize RPP1/ ATR1 complexes in order to evaluate and verify these predictions. Isolating soluble full-length NLR for such experiments has been notoriously difficult. However, in planta overexpression of the LRR protein polygalacturonase inhibitor protein (PGIP) produced sufficient quantities for crystallizing the protein, and its structure was subsequently used to identify residues important for binding fungal polygalacturonase (Di Matteo et al. 2003; Casasoli et al. 2009).

\section{CONCLUSION}

In summary, we combine natural variation and mutagenesis with structural information and prediction to gain insight into the basis of effector recognition. Experiments are currently underway to verify the importance of $\beta$-sheet polymorphisms and to use the system to study NLR protein activation. We present a model for 

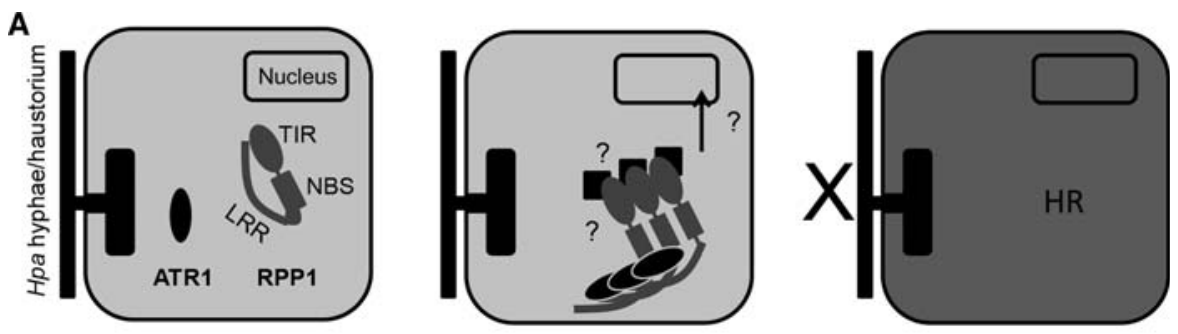
Recognized ATR1
delivery by Hpa (or surrogate system)

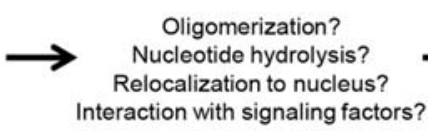

Hypersensitive response Hpa resistance

B
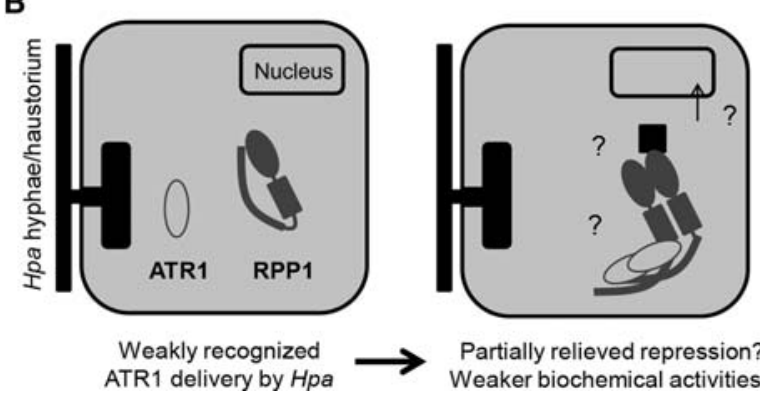

Partially relieved repression? Weaker biochemical activities?

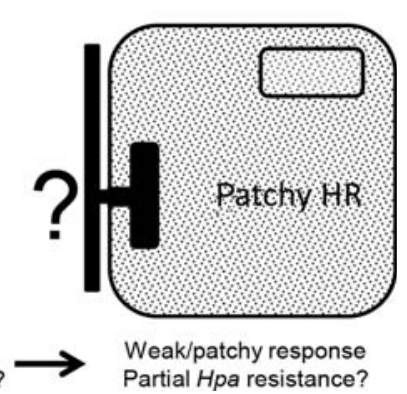

Figure 4. Model for NLR activation by fully recognized and weakly recognized effector alleles. (A) An incompatible Hpa strain delivers a fully recognized ATR1 allele, leading to strong outputs (oligomerization, nucleotide hydrolysis, translocation, and host factor interaction) that lead to resistance. $(B)$ Weakly recognized ATR1 alleles may lead to an attenuated or different combination of signaling outputs, leading to the patchy HR phenotype observed in surrogate assays. Weak LRR binding may be the basis for partial phenotypes observed with several ATR1 mutants and may be the basis for semicompatible strains of Hpa observed in natural strainaccession combinations.

recognition, activation, and signaling events in Figure 4. It is possible that delivery of a recognized allele of ATR1 triggers a number of steps shown to be critical for other NLRs: oligomerization, relocalization, and host factor binding. We direct readers to recent comprehensive reviews on these aspects of NLR function (Eitas and Dangl 2010; Heidrich et al. 2012; Takken and Goverse 2012). This cascade of events leads to a robust HR response and resistance (Fig. 4A). It will be interesting to compare and contrast RPP1 activation mechanisms with other plant NLR model systems that have already shown diverse modes of function. For the ATR1-RPP1 system specifically, we note the unique availability of several allelic combinations that trigger weak HR phenotypes in tobacco coexpression assays (e.g., the gain-of-recognition mutations imparting recognition by RPP1-WsB [Chou et al. 2011]). Activation mediated by these "weak" allelic combinations may allow us to observe a spectrum of resistance responses, providing a more nuanced view of NLR receptor activation than the traditional gene-for-gene model, where successful recognition triggers a strictly binary switch between "off" and "on" states. For example, RPP1 activation by a more weakly recognized ATR1 allele could trigger partial or patchy signaling outcomes downstream (Fig. 4B). Ultimately, thoroughly understanding the structural basis of NLR activation by effectors will be critical for manipulating these fascinating receptor proteins to engineer durable resistance in crop plants.

\section{ACKNOWLEDGMENTS}

We thank Rebecca Bart for critically reading this manuscript. Work is supported by National Science Foundation grant number IOS 1146793 to B.J.S.

\section{REFERENCES}

Baxter L, Tripathy S, Ishaque N, Boot N, Cabral A, Kemen E, Thines M, Ah-Fong A, Anderson R, Badejoko W, et al. 2010. Signatures of adaptation to obligate biotrophy in the Hyaloperonospora arabidopsidis genome. Science 330: 15491551.

Bella J, Hindle KL, McEwan PA, Lovell SC. 2008. The leucinerich repeat structure. Cell Mol Life Sci 65: 2307-2333.

Bernoux M, Ve T, Williams S, Warren C, Hatters D, Valkov E, Zhang X, Ellis JG, Kobe B, Dodds PN. 2011. Structural and functional analysis of a plant resistance protein TIR domain reveals interfaces for self-association, signaling, and autoregulation. Cell Host Microbe 9: 200-211.

Boller T, Felix G. 2009. A renaissance of elicitors: Perception of microbe-associated molecular patterns and danger signals by pattern-recognition receptors. Annu Rev Plant Biol 60: 379-406.

Botella MA, Parker JE, Frost LN, Bittner-Eddy PD, Beynon JL, Daniels MJ, Holub EB, Jones JDG. 1998. Three genes of the Arabidopsis RPP1 complex resistance locus recognize distinct Peronospora parasitica avirulence determinants. Plant Cell 10: 1847-1860.

Cabral A, Stassen JHM, Seidl MF, Bautor J, Parker JE, Van den Ackerveken G. 2011. Identification of Hyaloperonospora arabidopsidis transcript sequences expressed during infection reveals isolate-specific effectors. PloS One 6: e19328. 
Casasoli M, Federici L, Spinelli F, Di Matteo A, Vella N, Scaloni F, Fernandez-Recio J, Cervone F, De Lorenzo G. 2009. Integration of evolutionary and desolvation energy analysis identifies functional sites in a plant immunity protein. Proc Natl Acad Sci 106: 7666-7671.

Catanzariti A-M, Dodds PN, Ve T, Kobe B, Ellis JG, Staskawicz BJ. 2009. The AvrM effector from flax rust has a structured Cterminal domain and interacts directly with the $\mathrm{M}$ resistance protein. Mol Plant Microbe Interact 23: 49-57.

Chen R, Li L, Weng ZP. 2003. ZDOCK: An initial-stage proteindocking algorithm. Prot Struct Funct Genet 52: 80-87.

Chinchilla D, Bauer Z, Regenass M, Boller T, Felix G. 2006. The Arabidopsis receptor kinase FLS2 binds flg22 and determines the specificity of flagellin perception. Plant Cell 18: $465-476$.

Chisholm ST, Coaker G, Day B, Staskawicz BJ. 2006. Hostmicrobe interactions: Shaping the evolution of the plant immune response. Cell 124: 803-814.

Chou S, Krasileva KV, Holton JM, Steinbrenner AD, Alber T, Staskawicz BJ. 2011. Hyaloperonospora arabidopsidis ATR1 effector is a repeat protein with distributed recognition surfaces. Proc Natl Acad Sci 108: 13323-13328.

Cunnac S, Chakravarthy S, Kvitko BH, Russell AB, Martin GB, Collmer A. 2011. Genetic disassembly and combinatorial reassembly identify a minimal functional repertoire of type III effectors in Pseudomonas syringae. Proc Natl Acad Sci 108: 2975-2980.

Day B, Dahlbeck D, Huang J, Chisholm ST, Li D, Staskawicz BJ. 2005. Molecular basis for the RIN4 negative regulation of RPS2 disease resistance. Plant Cell 17: 1292-1305.

Dickson KA, Haigis MC, Raines RT. 2005. Ribonuclease inhibitor: Structure and function. In Prog Nucleic Acid Res Mol Biol (ed. Kivie M), pp. 349-374. Academic Press, NY.

Di Matteo A, Federici L, Mattei B, Salvi G, Johnson KA, Savino C, De Lorenzo G, Tsernoglou D, Cervone F. 2003. The crystal structure of polygalacturonase-inhibiting protein (PGIP), a leucine-rich repeat protein involved in plant defense. Proc Natl Acad Sci 100: 10124-10128.

Dodds PN, Rathjen JP. 2010. Plant immunity: Towards an integrated view of plant-pathogen interactions. Nat Rev Genet 11: $539-548$.

Dodds PN, Lawrence GJ, Catanzariti AM, Teh T, Wang CIA, Ayliffe MA, Kobe B, Ellis JG. 2006. Direct protein interaction underlies gene-for-gene specificity and coevolution of the flax resistance genes and flax rust avirulence genes. Proc Natl Acad Sci 103: 8888-8893.

Eitas TK, Dangl JL. 2010. NB-LRR proteins: Pairs, pieces, perception, partners, and pathways. Curr Opin Plant Biol 13: $472-477$.

Ellis JG, Lawrence GJ, Luck JE, Dodds PN. 1999. Identification of regions in alleles of the flax rust resistance gene $\mathrm{L}$ that determine differences in gene-for-gene specificity. Plant Cell 11: 495-506.

Fabro G, Steinbrenner J, Coates M, Ishaque N, Baxter L, Studholme DJ, Körner E, Allen RL, Piquerez SJM, Rougon-Cardoso A, et al. 2011. Multiple candidate effectors from the oomycete pathogen Hyaloperonospora arabidopsidis suppress host plant immunity. PLoS Pathog 7: e1002348.

Farnham G, Baulcombe DC. 2006. Artificial evolution extends the spectrum of viruses that are targeted by a disease-resistance gene from potato. Proc Natl Acad Sci 103: $18828-$ 18833.

Gordon A. 2002. Analysis of the RPP1 resistance gene cluster in Arabidopsis accession niederzenz (Nd-1). PhD thesis, University of Birmingham, Birmingham, UK.

Goritschnig S, Krasileva KV, Dahlbeck D, Staskawicz BJ. 2012. Computational prediction and molecular characterization of an oomycete effector and the cognate Arabidopsis resistance gene. PLoS Genet 8: e1002502.

Gunn ND, Byrne J, Holub EB. 2002. Outcrossing of two homothallic isolates of Peronospora parasitica and segregation of avirulence matching six resistance loci in Arabidopsis thaliana. In Advances in Downy Mildew Research (ed. Spencer-
Phillips PTN, Gisi U, Lebeda A), 185-188. Springer, Netherlands.

Heidrich K, Blanvillain-Baufumé S, Parker JE. 2012. Molecular and spatial constraints on NB-LRR receptor signaling. Curr Opin Plant Biol 15: 385-391.

Helft L, Reddy V, Chen X, Koller T, Federici L, FernándezRecio J, Gupta R, Bent A. 2011. LRR conservation mapping to predict functional sites within protein leucine-rich repeat domains. PloS One 6: e21614.

Holub EB, Beynon JL.1997. Symbiology of mouse-ear cress (Arabidopsis thaliana) and oomycetes. Adv Botan Res 24: 227-273.

Inohara N, Chamaillard M, McDonald C, Nuñez G. 2005. NOD-LRR PROTEINS: Role in host-microbial interactions and inflammatory disease. Annu Rev Biochem 74: 355-383.

Jia Y, McAdams SA, Bryan GT, Hershey HP, Valent B. 2000. Direct interaction of resistance gene and avirulence gene products confers rice blast resistance. EMBO J 19: 40044014

Johnson RJ, McCoy JG, Bingman CA, Phillips GN Jr, Raines RT. 2007. Inhibition of human pancreatic ribonuclease by the human ribonuclease inhibitor protein. J Mol Biol 368: $434-$ 449.

Jones JD, Dangl JL. 2006. The plant immune system. Nature 444: $323-329$.

Kale SD, Tyler BM. 2011. Assaying effector function in planta using double-barreled particle bombardment. Methods Mol Biol 712: $153-172$.

Kim H-S, Desveaux D, Singer AU, Patel P, Sondek J, Dangl JL. 2005. The pseudomonas syringae effector AvrRpt 2 cleaves its C-terminally acylated target, RIN4, from Arabidopsis membranes to block RPM1 activation. Proc Natl Acad Sci 102: 6496-6501.

Kobe B, Deisenhofer J. 1993. Crystal structure of porcine ribonuclease inhibitor, a protein with leucine-rich repeats. Nature 366: $751-756$.

Krasileva KV, Dahlbeck D, Staskawicz BJ. 2010. Activation of an Arabidopsis resistance protein is specified by the in planta association of its leucine-rich repeat domain with the cognate oomycete effector. Plant Cell 22: 2444-2458.

Krasileva KV, Zheng C, Leonelli L, Goritschnig S, Dahlbeck D, Staskawicz BJ. 2011. Global analysis of Arabidopsis/downy mildew interactions reveals prevalence of incomplete resistance and rapid evolution of pathogen recognition. PloS One 6: e28765.

Lee S-W, Han S-W, Sririyanum M, Park C-J, Seo Y-S, Ronald PC. 2009. A type I-secreted, sulfated peptide triggers XA21mediated innate immunity. Science 326: 850-853.

Leonelli L, Pelton J, Schoeffler A, Dahlbeck D, Berger J, Wemmer DE, Staskawicz B. 2011. Structural elucidation and functional characterization of the Hyaloperonospora arabidopsidis effector protein ATR13. PLoS Pathog 7: e1002428.

Lukasik E, Takken FLW. 2009. STANDing strong, resistance proteins instigators of plant defence. Curr Opin Plant Biol 12: $427-436$.

Mackey D, Holt BF, Wiig A, Dangl JL. 2002. RIN4 interacts with Pseudomonas syringae type III effector molecules and is required for RPM1-mediated resistance in Arabidopsis. Cell 108: $743-754$.

Oh C-S, Martin GB. 2011. Effector-triggered immunity mediated by the Pto kinase. Trends Plant Sci 16: 132-140.

Parker JE, Coleman MJ, Szado V, Frost LN, Schmidt R. van der Biezen EA, Moores T, Dean C, Daniels MJ, Jones JD. 1997. The Arabidopsis downy mildew resistance gene RPP5 shares similarity to the Toll and interleukin-1 receptors with $\mathrm{N}$ and L6. Plant Cell 9: 879-894.

Rehmany AP, Gordon A, Rose LE, Allen RL, Armstrong MR, Whisson SC, Kamoun S, Tyler BM, Birch PRJ, Beynon JL. 2005. Differential recognition of highly divergent downy mildew avirulence gene alleles by RPP1 resistance genes from two Arabidopsis lines. Plant Cell 17: 1839-1850.

Rentel MC, Leonelli L, Dahlbeck D, Zhao B, Staskawicz BJ. 2008. Recognition of the Hyaloperonospora parasitica 
effector ATR13 triggers resistance against oomycete, bacterial, and viral pathogens. Proc Natl Acad Sci 105: 1091-1096.

Sela H, Spiridon LN, Petrescu A-J, Akerman M, Mandel-Gutfreund Y, Nevo E, Loutre C, Keller B, Schulman AH, Fahima T. 2012. Ancient diversity of splicing motifs and protein surfaces in the wild emmer wheat (Triticum dicoccoides) LR10 coiled coil (CC) and leucine-rich repeat (LRR) domains. Mol Plant Pathol 13: 276-287.

Sohn KH, Lei R, Nemri A, Jones JDG. 2007. The downy mildew effector proteins ATR1 and ATR13 promote disease susceptibility in Arabidopsis thaliana. Plant Cell 19: $4077-$ 4090.

Takken FLW, Goverse A. 2012. How to build a pathogen detector: Structural basis of NB-LRR function. Curr Opin Plant Biol 15: 375-384.
Van der Hoorn RAL, Roth R, De Wit PJGM. 2001. Identification of distinct specificity determinants in resistance protein Cf-4 allows construction of a Cf-9 mutant that confers recognition of avirulence protein AVR4. Plant Cell 13: $273-285$.

Vleeshouwers VGAA, Rietman H, Krenek P, Champouret N, Young C, Oh S-K, Wang M, Bouwmeester K, Vosman B, Visser RGF, et al. 2008. Effector genomics accelerates discovery and functional profiling of potato disease resistance and Phytophthora infestans avirulence genes. PloS One 3: e2875.

Zipfel C, Kunze G, Chinchilla D, Caniard A, Jones JDG, Boller T, Felix G. 2006. Perception of the bacterial PAMP EF-Tu by the receptor EFR restricts Agrobacterium-mediated transformation. Cell 125: 749-760. 


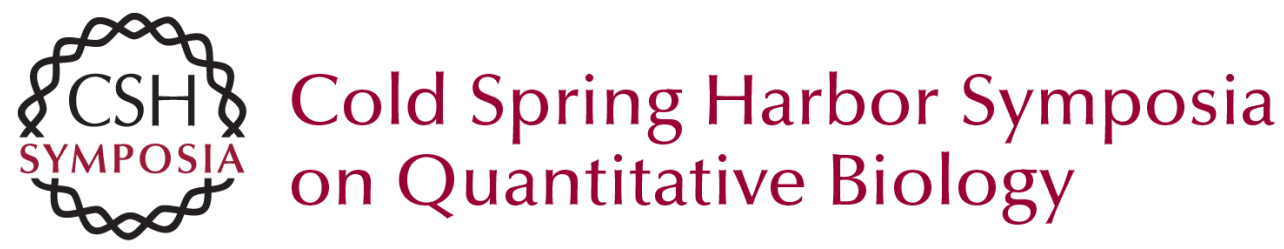

\section{Effector Recognition and Activation of the Arabidopsis thaliana NLR Innate Immune Receptors}

A.D. Steinbrenner, S. Goritschnig, K.V. Krasileva, et al.

Cold Spring Harb Symp Quant Biol 2012 77: 249-257 originally published online November 21, 2012

Access the most recent version at doi:10.1101/sqb.2012.77.014860

References This article cites 48 articles, 20 of which can be accessed free at: http://symposium.cshlp.org/content/77/249.full.html\#ref-list-1

\section{License}

Email Alerting Receive free email alerts when new articles cite this article - sign up in Service the box at the top right corner of the article or click here. 